\title{
Effect of Seed Size and Cutting Methods on the Yield and Profitability of Potato
}

\author{
M. S. Hossain ${ }^{1}$, M. Zakaria ${ }^{2}$, M. M. Hossain ${ }^{2}$ and M. H. Rashid ${ }^{3}$ \\ ${ }^{1}$ Seed Marketing Division, BADC, Gazipur, Bangladesh \\ ${ }^{2}$ Dept. of Horticulture, BSMRAU, Gazipur-1706, Bangladesh \\ ${ }^{4}$ Wheat Research Centre, Bangladesh Agricultural Research Institute, Dinajpur, Bangladesh
}

Corresponding author and Email: shafayetbadc@gmail.com

Received: 05 May 2011

Accepted: 26 November 2011

\begin{abstract}
An experiment was conducted at the research field of Tuber Crop Research Centre (TCRC) of Bangladesh Agricultural Research Institute, Gazipur during the winter of 2006-2007 to determine the effects of cutting methods and seed size on the yield and profitability in potato (Solanum tuberosum L.). It was observed that the foliage coverage, plant height, stems/hill, number and weight of tubers/plant varied significantly due to variation of tuber size. The highest germination index (18.13) was found when large size whole tuber was used followed by small size whole tuber and medium size whole tuber. Vigorous plants, maximum plant height and the number of stems/ hill at 60 DAP were also produced when the crop was raised from large size whole tubers. The maximum leaf area of 3909 $\mathrm{cm}^{2}$ was found in medium size -half proximal treatment. The highest number (10.23) and weight of tubers/ hill $(0.40 \mathrm{~kg})$ were observed in $\mathrm{T}_{6}$ large size-whole tuber treatment. The highest gross yield of tubers $(25.10 \mathrm{t} / \mathrm{ha})$ was also produced when large whole tubers were used. However, small tuber size and half cut distal would be economically profitable for enhancing growth and yield of potato.
\end{abstract}

\section{Keywords: Potato, seed size, cutting methods, profitability}

\section{Introduction}

The average yield of potato (Solanum tuberosum) is about $14.76 \mathrm{t} / \mathrm{ha}$ in Bangladesh which is very low compared to the other leading potato growing countries like Belgium, New Zealand, the Netherlands and United Kingdom (Chadla et al., 2001). Now it occupies the second place in acreage and production after rice (Anonymous, 2010). The potential yield of the crop is much higher than the average yield. Among the many causes of low yield of potato, lack of improved production technologies is very important. Seed quality, seed size, seed cutting, plant spacing etc. are some of the management practices which should be standardized for each agro-climatic zones of the country. At present, the seed supply from recognizable sources is about 19,000 metric tons. Out of the total requirement, $\mathrm{BADC}$ is supplying only about $2 \%$ that is used by the farmers as the replacement stock (Talukder, 2004). The cost of seed, or in other words the seed rate is directly associated with the size of seed and plant spacing. On the other hand, the yield of potato is influenced by the size of seed. In general larger seed and closer planting up to a certain limit increase the yield of tubers per unit area. Khurana et al. (1994) suggested that for a given seed tuber size, total tuber yield increased with increase in tuber rate. For a given seed tuber rate $(2.5 \mathrm{t} / \mathrm{ha})$, total seed tuber yield decreased with increase in seed tuber size. The cost of seed potato is very high and it constitutes from 25 to $50 \%$ of the total cost of production in Bangladesh. For reducing the seed 
cost farmers of many potato-growing areas are using cut tubers with closer spacing although TCRC recommended whole tuber with $60 \mathrm{~cm} \mathrm{X}$ $25 \mathrm{~cm}$ spacing. However, seed cost remains more or less same as the cost of using whole tuber at wider spacing, since the farmers use closer spacing for cut seed. Many researchers also found better yield by using cut seed with closer spacing (Bushan, 2007; Zebarth, 2006; Garba, 2005; Shaha, 2004; Hoque, 2001; Nandekar et al., 1993; Banarjee et al., 1988). In case of cut tuber, spacing should be the same as recommended for whole tuber to reduce the seed cost. In this context, an investigation was undertaken to find out proper combination of seed size and cutting methods for profitable potato production

\section{Materials and Methods}

The investigation was carried out at the research field of Tuber Crops Research Centre (TCRC) of Bangladesh Agricultural Research Institute (BARI), Gazipur during winter, 2006-2007. All sizes of tubers were cut longitudinally to obtain longitudinal half cut. Longitudinal half cut pieces were cut again for getting $1 / 4^{\text {th }}$ longitudinal cut. All sizes of tubers were cut horizontally (cross wise) to obtain half distal and half proximal cut pieces. Half distal and half proximal cut pieces were cut into half for obtaining $1 / 4^{\text {th }}$ crosswise cut pieces. Thus, longitudinal half cut, $1 / 4^{\text {th }}$ longitudinal cut, distal half cut, proximal half cut and $1 / 4^{\text {th }}$ cut crosswise pieces of all sizes tubers were obtained. Seed size ' $\mathrm{S}$ ' (Large- $\mathrm{S}_{1}=45-55 \mathrm{~g}$, Medium- $\mathrm{S}_{2}=35-45$ $\mathrm{g}$, Small- $\mathrm{S}_{3}=25-35 \mathrm{~g}$ and cutting methods ' $\mathrm{C}$ ' (Half longitudinal- $\mathrm{C}_{1}$, Half distal- $\mathrm{C}_{2}$, Half proximal- $\mathrm{C}_{3}, \quad 1 / 4^{\text {th }} \quad$ crosswise- $\mathrm{C}_{4}, \quad 1 / 4^{\text {th }}$ longitudinal- $\mathrm{C}_{5}$, Whole tuber- $\mathrm{C}_{6}$ ) were the two factors. The three sizes of seed tubers and six cutting methods made 18 treatments. The treatments were designated as $\mathrm{T}_{1}=\mathrm{S}_{1} \mathrm{C}_{1}=$ Large size-Half longitudinal, $\mathrm{T}_{2}=\mathrm{S}_{1} \mathrm{C}_{2}=$ Large size-Half distal, $\mathrm{T}_{3}=\mathrm{S}_{1} \mathrm{C}_{3}=$ Large size-Half proximal, $\mathrm{T}_{4}=\mathrm{S}_{1} \mathrm{C}_{4}=$ Large size- $1 / 4^{\text {th }}$ crosswise, $\mathrm{T}_{5}=\mathrm{S}_{1} \mathrm{C}_{5}$ $=$ Large size- $1 / 4^{\text {th }}$ longitudinal, $\mathrm{T}_{6}=\mathrm{S}_{1} \mathrm{C}_{6}=$ Large size-Whole tuber, $\mathrm{T}_{7}=\mathrm{S}_{2} \mathrm{C}_{1}=$ Medium size-Half longitudinal, $\mathrm{T}_{8}=\mathrm{S}_{2} \mathrm{C}_{2}=$ Medium size-Half distal,
$\mathrm{T}_{9}=\mathrm{S}_{2} \mathrm{C}_{3}=$ Medium size- Half proximal, $\mathrm{T}_{10}=\mathrm{S}_{2} \mathrm{C}_{4}=$ Medium size- $1 / 4^{\text {th }}$ crosswise, $\mathrm{T}_{11}=\mathrm{S}_{2} \mathrm{C}_{5}=$ Medium size- $1 / 4^{\text {th }}$ longitudinal, $\mathrm{T}_{12}=\mathrm{S}_{2} \mathrm{C}_{6}=$ Medium size-Whole tuber, $\mathrm{T}_{13}=\mathrm{S}_{3} \mathrm{C}_{1}=$ Small size-Half longitudinal, $\mathrm{T}_{14}=\mathrm{S}_{3} \mathrm{C}_{2}=$ Small size-Half distal, $\mathrm{T}_{15}=\mathrm{S}_{3} \mathrm{C}_{3}=$ Small size-Half proximal, $\mathrm{T}_{16}=\mathrm{S}_{3} \mathrm{C}_{4}=$ Small size- $1 / 4^{\text {th }}$ crosswise, $\mathrm{T}_{17}=\mathrm{S}_{3} \mathrm{C}_{5}=$ Small $\quad$ size $-1 / 4^{\text {th }} \quad$ longitudinal, $\mathrm{T}_{18}=\mathrm{S}_{3} \mathrm{C}_{6}=$ Small size-Whole tuber. The experiment was laid out in a randomized complete block design (RCBD) with three replications.

The seed tubers were planted in $3 \mathrm{~m}$ x $3 \mathrm{~m}$ plots on $28^{\text {th }}$ November 2006 maintaining a spacing of $60 \mathrm{~cm} \times 25 \mathrm{~cm}$. Thus each unit plot accommodated 60 pieces (whole or cut) in 5 rows. The depth of planting was $10 \mathrm{~cm}$ from the surface of the soil. Data were recorded on germination index, plant height, plant vigor (110 score), foliage coverage, no. of stems / hill, area of leaf $\left(\mathrm{cm}^{2}\right)$, no. of tubers / hill, wt. of tubers / hill, yield of tubers per plot, yield of tubers / hectare, tuber grade by number, tuber grade by weight.

Data on different parameters were analyzed statistically using MSTAT-C program to find out the significance of experimental result. Partial economic analysis was done in order to find out comparative benefits of the combination treatments of seed size and cutting methods. For this purpose cost for seed cutting, fertilizer / pesticides application and harvesting of tubers were recorded for unit plot and then converted into cost per hectare. Partial analysis was done calculating non-material (labour) and material (seed) input costs for computing the cost of production. The price of the potato tubers was determined on market price basis. At harvest it was considered to be Tk.7.00, 12.00, 17.00, 16.00 and 15.00 for tuber grade $<20 \mathrm{~mm}$ (under size), 20-28mm (under size), 28-40mm (A grade), $40-55 \mathrm{~mm}$ (B grade) and $>55 \mathrm{~mm}$ (over size), respectively (Table 5). Less than $20 \mathrm{~mm}$ and 20-28 $\mathrm{mm}$ size are considered as non seed in consideration of seed grade and less than $20 \mathrm{~mm}$ size tuber is considered as non-marketable seed 
potato. So, their price was minimum for under sized seed potato and maximum in seed sized potato (A and $\mathrm{B}$ ) in the harvesting season.

\section{Results and Discussion}

\subsection{Morphological characters and yield}

The data on morphological characters and yield are presented in Tables 1, 2 and 3. The effect of seed size and cutting methods on all the characters under study varied significantly. Maximum germination index (18.13) was observed in $\mathrm{T}_{6}$, while the lowest (8.71) was in $\mathrm{T}_{17}$. Plant vigor scored to be the highest in $\mathrm{T}_{6}$ at 30,45 and 60 days after planting, while the minimum (3.33) was in $\mathrm{T}_{16}$ and $\mathrm{T}_{17}$. Effect of seed size and cutting methods on foliage development was increased with the progress in DAP. Highest foliage coverage $(85.67 \%)$ was produced by $\mathrm{T}_{6}$ (large whole tuber) followed by $\mathrm{T}_{12}, \mathrm{~T}_{8}, \mathrm{~T}_{2}, \mathrm{~T}_{18}$ and $\mathrm{T}_{14}$, while the lowest (16.67\%) was in $\mathrm{T}_{17}$ (small $1 / 4^{\text {th }}$ cut longitudinal) (Table 1). The highest foliage coverage in the treatment $\mathrm{T}_{6}$ at 60 DAP might be due to more number of stems produced from the whole tuber that possessed more eyes. During the period of plant growth the maximum plant height was recorded from the treatment $\mathrm{T}_{6}(43.40 \mathrm{~cm})$ followed by $\mathrm{T}_{12}(36.20 \mathrm{~cm})$ while the minimum from $\mathrm{T}_{11}(22.87 \mathrm{~cm})$. Similar trend of plant height was also observed at 30 and 45 DAP.

Table 1. Effect of seed size and cutting methods on germination index, plant vigour and foliage coverage

\begin{tabular}{|c|c|c|c|c|c|c|c|}
\hline \multirow[t]{2}{*}{ Treatment } & \multirow{2}{*}{$\begin{array}{l}\text { Germination } \\
\text { index }\end{array}$} & \multicolumn{3}{|c|}{ Plant vigor (1-10) } & \multicolumn{3}{|c|}{ Foliage coverage $(\%)$} \\
\hline & & 30 DAP & 45 DAP & 60 DAP & 30 DAP & 45DAP & 60 DAP \\
\hline $\mathrm{T}_{1}=\mathrm{S}_{1} \mathrm{C}_{1}$ & $15.35 b c$ & $4.67 \mathrm{e}$ & $5.00 \mathrm{de}$ & $5.00 \mathrm{de}$ & 30.00de & $41.67 \mathrm{de}$ & $46.67 d$ \\
\hline $\mathrm{T}_{2}=\mathrm{S}_{1} \mathrm{C}_{2}$ & $15.97 b c$ & $6.67 b c$ & $6.67 \mathrm{c}$ & $7.33 b$ & $53.33 b c$ & $61.67 b c$ & $66.67 b c$ \\
\hline $\mathrm{T}_{3}=\mathrm{S}_{1} \mathrm{C}_{3}$ & $12.97 \mathrm{de}$ & $3.67 \mathrm{e}-\mathrm{h}$ & $4.00 \mathrm{fg}$ & $5.00 \mathrm{de}$ & $25.00 \mathrm{ef}$ & $31.67 \mathrm{fg}$ & $35.00 \mathrm{ef}$ \\
\hline $\mathrm{T}_{4}=\mathrm{S}_{1} \mathrm{C}_{4}$ & $12.48 \mathrm{e}$ & $4.67 \mathrm{de}$ & $5.67 \mathrm{~d}$ & $6.00 \mathrm{~cd}$ & $30.00 \mathrm{de}$ & 38.33 def & $41.67 \mathrm{de}$ \\
\hline $\mathrm{T}_{5}=\mathrm{S}_{1} \mathrm{C}_{5}$ & $12.38 \mathrm{e}$ & $3.67 \mathrm{e}-\mathrm{h}$ & $4.33 \mathrm{ef}$ & $4.67 \mathrm{de}$ & $31.67 \mathrm{de}$ & $35.00 \mathrm{ef}$ & 43.33de \\
\hline $\mathrm{T}_{6}=\mathrm{S}_{1} \mathrm{C}_{6}$ & $18.13 \mathrm{a}$ & $8.00 \mathrm{a}$ & $8.67 \mathrm{a}$ & $9.00 \mathrm{a}$ & $71.67 \mathrm{a}$ & $83.33 \mathrm{a}$ & $85.67 \mathrm{a}$ \\
\hline $\mathrm{T}_{7}=\mathrm{S}_{2} \mathrm{C}_{1}$ & $14.62 \mathrm{~cd}$ & $4.33 \mathrm{ef}$ & $5.33 d$ & $6.00 \mathrm{~cd}$ & $35.00 \mathrm{~d}$ & $41.67 \mathrm{de}$ & $48.33 \mathrm{~d}$ \\
\hline $\mathrm{T}_{8}=\mathrm{S}_{2} \mathrm{C}_{2}$ & $14.23 \mathrm{cde}$ & $7.67 \mathrm{ab}$ & $7.67 b$ & $7.67 \mathrm{~b}$ & $56.67 b c$ & $61.67 b c$ & $66.67 b c$ \\
\hline $\mathrm{T}_{9}=\mathrm{S}_{2} \mathrm{C}_{3}$ & $13.30 \mathrm{de}$ & $4.00 \mathrm{efg}$ & $5.67 \mathrm{~d}$ & $6.00 \mathrm{~cd}$ & $35.00 \mathrm{~d}$ & $41.67 \mathrm{de}$ & 43.33de \\
\hline $\mathrm{T}_{10}=\mathrm{S}_{2} \mathrm{C}_{4}$ & $10.65 f$ & 3.00ghi & $3.67 \mathrm{fg}$ & 4.00 ef & $21.67 \mathrm{fg}$ & $26.67 \mathrm{gh}$ & $26.67 \mathrm{fg}$ \\
\hline $\mathrm{T}_{11}=\mathrm{S}_{2} \mathrm{C}_{5}$ & $8.98 \mathrm{fg}$ & $2.00 \mathrm{i}$ & $2.67 \mathrm{~h}$ & $3.00 \mathrm{f}$ & $14.00 \mathrm{~h}$ & $18.33 \mathrm{ij}$ & $18.33 \mathrm{~g}$ \\
\hline $\mathrm{T}_{12}=\mathrm{S}_{2} \mathrm{C}_{6}$ & $16.67 \mathrm{ab}$ & $7.00 \mathrm{ab}$ & $7.00 \mathrm{bc}$ & $7.67 b$ & $60.00 \mathrm{~b}$ & $66.67 \mathrm{~b}$ & $73.33 \mathrm{~b}$ \\
\hline $\mathrm{T}_{13}=\mathrm{S}_{3} \mathrm{C}_{1}$ & $14.73 \mathrm{~cd}$ & $5.67 \mathrm{~cd}$ & $5.67 \mathrm{~d}$ & $5.67 d$ & $36.67 d$ & $43.33 d$ & 45.00de \\
\hline $\mathrm{T}_{14}=\mathrm{S}_{3} \mathrm{C}_{2}$ & $15.53 b c$ & $6.67 b c$ & $6.67 \mathrm{c}$ & $7.00 \mathrm{bc}$ & $50.00 \mathrm{c}$ & $55.00 \mathrm{c}$ & $61.67 \mathrm{c}$ \\
\hline $\mathrm{T}_{15}=\mathrm{S}_{3} \mathrm{C}_{3}$ & $15.33 b c$ & $3.67 \mathrm{e}-\mathrm{h}$ & $4.33 \mathrm{ef}$ & $5.33 d$ & $31.67 \mathrm{de}$ & $36.67 \mathrm{def}$ & $43.33 \mathrm{de}$ \\
\hline $\mathrm{T}_{16}=\mathrm{S}_{3} \mathrm{C}_{4}$ & $10.40 f g$ & $2.67 \mathrm{hi}$ & $3.33 \mathrm{gh}$ & $3.33 \mathrm{f}$ & $16.67 \mathrm{gh}$ & $21.67 \mathrm{hi}$ & $21.67 \mathrm{~g}$ \\
\hline $\mathrm{T}_{17}=\mathrm{S}_{3} \mathrm{C}_{5}$ & $8.72 \mathrm{~g}$ & $3.33 \mathrm{fgh}$ & $3.33 \mathrm{gh}$ & $3.33 \mathrm{f}$ & $13.33 \mathrm{~h}$ & $13.33 \mathrm{j}$ & $16.67 \mathrm{~g}$ \\
\hline $\mathrm{T}_{18}=\mathrm{S}_{3} \mathrm{C}_{6}$ & $17.13 \mathrm{ab}$ & $6.67 \mathrm{bc}$ & $6.67 \mathrm{c}$ & $7.00 \mathrm{bc}$ & $56.67 \mathrm{bc}$ & $60.00 \mathrm{bc}$ & $63.33 b c$ \\
\hline $\mathrm{CV} \%$ & 7.45 & 11.75 & 9.77 & 12.19 & 11.00 & 9.62 & 12.71 \\
\hline $\begin{array}{l}\text { Level of } \\
\text { significanc }\end{array}$ & $* *$ & $*$ & $*$ & $*$ & $*$ & $*$ & $*$ \\
\hline
\end{tabular}

$* \& * *$ indicate significance at $5 \%$ \& $1 \%$ level of probability, DAP= Days after planting. Means in a column followed by the same letters do not differ significantly at $1 \%$ level of significance. ' $\mathrm{S}$ ' and ' $\mathrm{C}$ ' indicate seed size (large- $\mathrm{S}_{1}$, medium- $\mathrm{S}_{2}$, and small- $\mathrm{S}_{3}$ ) and cutting methods (half longitudinal- $\mathrm{C}_{1}$, half distal- $\mathrm{C}_{2}$, half proximal- $\mathrm{C}_{3}, 1 / 4^{\text {th }}$ crosswise- $\mathrm{C}_{4}, 1 / 4^{\text {th }}$ longitudinal- $\mathrm{C}_{5}$, whole tuber- $\mathrm{C}_{6}$ ), respectively 
The maximum number of stems was found in $\mathrm{T}_{6}$ (8.4). The second highest number of stems per hill (5.93) was produced in $\mathrm{T}_{12}$ (medium sized whole tuber), which was statistically similar to $\mathrm{T}_{2}$ (large half cut distal). The minimum number of stems per hill was in $\mathrm{T}_{11}$ (1.6). Area of leaf varied significantly among the treatment combinations ranging from 2215 to $3909 \mathrm{~cm}^{2}$
(Table 2). The maximum area of leaf was found in $\mathrm{T}_{9}$ and the minimum was observed in $\mathrm{T}_{14}$. The highest number of tubers/hill was observed in the treatment $\mathrm{T}_{6}$ (10.23). Half cut distal of large, medium and small size tubers $\left(T_{2}, T_{8}\right.$ and $\left.T_{14}\right)$ performed better among the different treatment combinations next to the treatments containing whole tuber.

Table 2. Interaction effects of seed size and cutting methods on plant height, stem per hill and leaf area

\begin{tabular}{|c|c|c|c|c|c|}
\hline \multirow{2}{*}{ Treatment } & \multicolumn{3}{|c|}{ Plant height $(\mathrm{cm})$} & \multirow{2}{*}{$\begin{array}{c}\text { No. of stem } \\
\text { /hill }\end{array}$} & \multirow{2}{*}{$\begin{array}{c}\text { Area of leaf } \\
\left(\mathrm{cm}^{2}\right)\end{array}$} \\
\hline & 30 DAP & 45 DAP & 60 DAP & & \\
\hline $\mathrm{T}_{1}=\mathrm{S}_{1} \mathrm{C}_{1}$ & $13.50 \mathrm{gh}$ & $24.33 \mathrm{bcd}$ & $30.77 \mathrm{~b}-\mathrm{e}$ & $3.60 \mathrm{de}$ & $3786 a b$ \\
\hline $\mathrm{T}_{2}=\mathrm{S}_{1} \mathrm{C}_{2}$ & $18.87 \mathrm{bc}$ & $29.10 \mathrm{ab}$ & $36.13 \mathrm{bcd}$ & $5.43 \mathrm{~b}$ & $3609 a b c$ \\
\hline $\mathrm{T}_{3}=\mathrm{S}_{1} \mathrm{C}_{3}$ & $16.13 \mathrm{def}$ & $27.87 \mathrm{bc}$ & $32.97 \mathrm{bcd}$ & $4.67 \mathrm{c}$ & $3729 a b$ \\
\hline $\mathrm{T}_{4}=\mathrm{S}_{1} \mathrm{C}_{4}$ & $12.87 \mathrm{~h}$ & $26.53 \mathrm{bcd}$ & $32.20 \mathrm{~b}-\mathrm{e}$ & $3.33 \mathrm{e}$ & $3526 a b c$ \\
\hline $\mathrm{T}_{5}=\mathrm{S}_{1} \mathrm{C}_{5}$ & $13.53 \mathrm{gh}$ & $26.13 \mathrm{bcd}$ & $29.37 \mathrm{def}$ & $3.33 \mathrm{e}$ & $3129 \mathrm{bcd}$ \\
\hline $\mathrm{T}_{6}=\mathrm{S}_{1} \mathrm{C}_{6}$ & $21.77 \mathrm{a}$ & $33.30 \mathrm{a}$ & $43.40 \mathrm{a}$ & $8.40 \mathrm{a}$ & 3241 a-d \\
\hline $\mathrm{T}_{7}=\mathrm{S}_{2} \mathrm{C}_{1}$ & $15.63 \mathrm{~d}-\mathrm{g}$ & $26.53 \mathrm{bcd}$ & $33.07 \mathrm{bcd}$ & 3.00 ef & 3259 a-d \\
\hline $\mathrm{T}_{8}=\mathrm{S}_{2} \mathrm{C}_{2}$ & $16.80 \mathrm{cde}$ & $27.73 \mathrm{bc}$ & $32.70 \mathrm{bcd}$ & $4.33 \mathrm{~cd}$ & $2765 \mathrm{de}$ \\
\hline $\mathrm{T}_{9}=\mathrm{S}_{2} \mathrm{C}_{3}$ & $12.83 \mathrm{~h}$ & $25.20 \mathrm{bcd}$ & $31.17 \mathrm{~b}-\mathrm{e}$ & $3.13 \mathrm{ef}$ & 3909 a \\
\hline $\mathrm{T}_{10}=\mathrm{S}_{2} \mathrm{C}_{4}$ & $10.37 \mathrm{i}$ & $21.43 \mathrm{de}$ & $26.93 \mathrm{efg}$ & $2.13 \mathrm{gh}$ & 2923 cde \\
\hline $\mathrm{T}_{11}=\mathrm{S}_{2} \mathrm{C}_{5}$ & $9.70 \mathrm{i}$ & $14.37 \mathrm{f}$ & $22.87 \mathrm{~g}$ & $1.60 \mathrm{~h}$ & $2760 \mathrm{de}$ \\
\hline $\mathrm{T}_{12}=\mathrm{S}_{2} \mathrm{C}_{6}$ & $20.50 \mathrm{ab}$ & $29.43 \mathrm{ab}$ & $36.20 \mathrm{~b}$ & $5.93 \mathrm{~b}$ & $2684 \mathrm{de}$ \\
\hline $\mathrm{T}_{13}=\mathrm{S}_{3} \mathrm{C}_{1}$ & $14.23 \mathrm{fgh}$ & $25.10 \mathrm{bcd}$ & 30.17 cde & 3.00 ef & $2328 \mathrm{e}$ \\
\hline $\mathrm{T}_{14}=\mathrm{S}_{3} \mathrm{C}_{2}$ & $15.40 \mathrm{efg}$ & $27.00 \mathrm{bcd}$ & $35.17 \mathrm{bc}$ & $3.40 \mathrm{e}$ & $2215 \mathrm{e}$ \\
\hline $\mathrm{T}_{15}=\mathrm{S}_{3} \mathrm{C}_{3}$ & $12.67 \mathrm{~h}$ & $23.30 \mathrm{cde}$ & $32.00 \mathrm{~b}-\mathrm{e}$ & $2.53 \mathrm{fg}$ & $3526 \mathrm{abc}$ \\
\hline $\mathrm{T}_{16}=\mathrm{S}_{3} \mathrm{C}_{4}$ & $10.07 \mathrm{i}$ & $16.20 \mathrm{f}$ & $24.57 \mathrm{fg}$ & $1.80 \mathrm{gh}$ & $2558 \mathrm{de}$ \\
\hline $\mathrm{T}_{17}=\mathrm{S}_{3} \mathrm{C}_{5}$ & $10.20 \mathrm{i}$ & 18.33 ef & $28.67 \mathrm{def}$ & $1.67 \mathrm{~h}$ & $2309 \mathrm{e}$ \\
\hline $\mathrm{T}_{18}=\mathrm{S}_{3} \mathrm{C}_{6}$ & $18.03 \mathrm{~cd}$ & $26.03 \mathrm{bcd}$ & $33.97 \mathrm{bcd}$ & $4.27 \mathrm{~cd}$ & $3125 \mathrm{bcd}$ \\
\hline $\mathrm{CV} \%$ & 9.30 & 11.71 & 9.12 & 11.63 & 12.16 \\
\hline $\begin{array}{l}\text { Level of } \\
\text { significance }\end{array}$ & $*$ & $*$ & $*$ & $*$ & $*$ \\
\hline
\end{tabular}

*, Significant at $5 \%$ level of probability. Means in a column followed by the same letters do not differ significantly at $5 \%$ level of significance. ' $\mathrm{S}$ ' and ' $\mathrm{C}$ ' indicate seed size (large- $\mathrm{S}_{1}$, medium- $\mathrm{S}_{2}$, and small- $\mathrm{S}_{3}$ ) and cutting methods (half longitudinal- $\mathrm{C}_{1}$, half distal- $\mathrm{C}_{2}$, half proximal $-\mathrm{C}_{3}, 1 / 4^{\text {th }}$ crosswise$\mathrm{C}_{4}, 1 / 4^{\text {th }}$ longitudinal- $\mathrm{C}_{5}$, whole tuber- $\mathrm{C}_{6}$ ), respectively 
The maximum weight of tubers/hill was found in $\mathrm{T}_{6}(0.40 \mathrm{Kg})$. The yield of tubers/hill was minimum $(0.19 \mathrm{~kg})$ in $\mathrm{T}_{16}$. The highest yield of tubers $\left(25.10 \mathrm{t} \mathrm{ha}^{-1}\right.$ ) was produced in $\mathrm{T}_{6}$ (large whole tuber) closely followed by $\mathrm{T}_{12}$ (medium size-whole tuber=23.01 t), $\mathrm{T}_{2}$ (large size-half distal=22.13 $\mathrm{t}$ ), $\quad \mathrm{T}_{18} \quad$ (small size-whole tuber=20.93 t) (Table 3). The results indicated that half distal cut of large sized tubers are comparable to small and medium sized whole tuber, which might be due to more or less equal number of eyes in both cut piece of large sized tubers and whole tuber of small and medium sized. The maximum percentage of tubers of $<20 \mathrm{~mm}, 20-28 \mathrm{~mm}, 28-40 \mathrm{~mm}, 40-55 \mathrm{~mm}$ and $>55 \mathrm{~mm}$ grades were 9.11, 22.43, 48.36, 47.58 and 8.18 in $\mathrm{T}_{5}, \mathrm{~T}_{12}, \mathrm{~T}_{2}, \mathrm{~T}_{11}$ and $\mathrm{T}_{15}$, respectively. It was found that $\mathrm{T}_{2}$ produced the maximum percentage $(48.36 \%)$ of $28-40 \mathrm{~mm}$ size tubers.

Table 3. Effects of seed size and cutting methods on number and weight of tubers per hill and yield of tubers / hectare

\begin{tabular}{|c|c|c|c|}
\hline Treatment & No. of tubers/hill & Wt.of tuber/hill( $(\mathrm{kg})$ & Yield (t/ha) \\
\hline $\mathrm{T}_{1}=\mathrm{S}_{1} \mathrm{C}_{1}$ & $5.77 \mathrm{~d}-\mathrm{g}$ & 0.32 cde & $17.74 \mathrm{c}$ \\
\hline $\mathrm{T}_{2}=\mathrm{S}_{1} \mathrm{C}_{2}$ & $8.07 \mathrm{~b}$ & $0.39 a b$ & $22.13 \mathrm{~b}$ \\
\hline $\mathrm{T}_{3}=\mathrm{S}_{1} \mathrm{C}_{3}$ & $6.37 \mathrm{cde}$ & $0.34 \mathrm{a}-\mathrm{d}$ & $15.60 \mathrm{~cd}$ \\
\hline $\mathrm{T}_{4}=\mathrm{S}_{1} \mathrm{C}_{4}$ & $6.30 \mathrm{c}-\mathrm{f}$ & $0.38 \mathrm{abc}$ & $16.71 \mathrm{c}$ \\
\hline $\mathrm{T}_{5}=\mathrm{S}_{1} \mathrm{C}_{5}$ & $5.80 \mathrm{~d}-\mathrm{g}$ & $0.34 \mathrm{a}-\mathrm{d}$ & $16.71 \mathrm{c}$ \\
\hline $\mathrm{T}_{6}=\mathrm{S}_{1} \mathrm{C}_{6}$ & $10.23 \mathrm{a}$ & $0.40 \mathrm{a}$ & $25.10 \mathrm{a}$ \\
\hline $\mathrm{T}_{7}=\mathrm{S}_{2} \mathrm{C}_{1}$ & $5.60 \mathrm{~d}-\mathrm{g}$ & $0.36 \mathrm{abc}$ & $15.69 \mathrm{~cd}$ \\
\hline $\mathrm{T}_{8}=\mathrm{S}_{2} \mathrm{C}_{2}$ & $6.93 \mathrm{c}$ & $0.34 \mathrm{a}-\mathrm{d}$ & $17.60 \mathrm{~cd}$ \\
\hline $\mathrm{T}_{9}=\mathrm{S}_{2} \mathrm{C}_{3}$ & 6.57 cde & $0.35 \mathrm{abc}$ & $16.21 \mathrm{~cd}$ \\
\hline $\mathrm{T}_{10}=\mathrm{S}_{2} \mathrm{C}_{4}$ & $5.70 \mathrm{~d}-\mathrm{g}$ & 0.32 cde & $9.96 \mathrm{f}$ \\
\hline $\mathrm{T}_{11}=\mathrm{S}_{2} \mathrm{C}_{5}$ & $4.03 \mathrm{hi}$ & $0.20 \mathrm{gh}$ & $7.60 \mathrm{~g}$ \\
\hline $\mathrm{T}_{12}=\mathrm{S}_{2} \mathrm{C}_{6}$ & $8.10 \mathrm{~b}$ & $0.39 \mathrm{ab}$ & $23.01 \mathrm{ab}$ \\
\hline $\mathrm{T}_{13}=\mathrm{S}_{3} \mathrm{C}_{1}$ & $5.23 \mathrm{fg}$ & $0.27 \mathrm{ef}$ & $14.13 \mathrm{de}$ \\
\hline $\mathrm{T}_{14}=\mathrm{S}_{3} \mathrm{C}_{2}$ & $6.97 \mathrm{c}$ & $0.38 \mathrm{abc}$ & $16.01 \mathrm{c}$ \\
\hline $\mathrm{T}_{15}=\mathrm{S}_{3} \mathrm{C}_{3}$ & $5.57 \mathrm{efg}$ & $0.29 \mathrm{def}$ & $12.96 \mathrm{e}$ \\
\hline $\mathrm{T}_{16}=\mathrm{S}_{3} \mathrm{C}_{4}$ & $3.80 \mathrm{i}$ & $0.19 \mathrm{~h}$ & $6.15 \mathrm{~g}$ \\
\hline $\mathrm{T}_{17}=\mathrm{S}_{3} \mathrm{C}_{5}$ & $4.93 \mathrm{gh}$ & $0.25 \mathrm{fg}$ & $6.53 \mathrm{~g}$ \\
\hline $\mathrm{T}_{18}=\mathrm{S}_{3} \mathrm{C}_{6}$ & $6.70 \mathrm{~cd}$ & $0.35 \mathrm{abc}$ & $20.93 \mathrm{~b}$ \\
\hline Level of significance & $*$ & $*$ & $*$ \\
\hline $\mathrm{CV} \%$ & 9.29 & 11.66 & 8.38 \\
\hline
\end{tabular}

* Significant at $5 \%$ level of probability. Means in a column followed by the same letter do not differ significantly at $5 \%$ level of probability. ' $\mathrm{S}$ ' and ' $\mathrm{C}$ ' indicate seed size (large- $\mathrm{S}_{1}$, medium- $\mathrm{S}_{2}$, and small- $\mathrm{S}_{3}$ ) and cutting methods (half longitudinal- $\mathrm{C}_{1}$, half distal- $\mathrm{C}_{2}$, half proximal- $\mathrm{C}_{3}, 1 / 4^{\text {th }}$ crosswise- $\mathrm{C}_{4}, 1 / 4^{\text {th }}$ longitudinal- $\mathrm{C}_{5}$, whole tuber- $\mathrm{C}_{6}$ ), respectively 
Table 4. Effects of seed size and cutting methods on tuber grade by weight (\%)

\begin{tabular}{|c|c|c|c|c|c|}
\hline \multirow{2}{*}{ Treatment } & \multicolumn{5}{|c|}{ Tuber grade by weight $(\%)$} \\
\hline & $<20 \mathrm{~mm}$ & $20-28 \mathrm{~mm}$ & $28-40 \mathrm{~mm}$ & $40-55 \mathrm{~mm}$ & $>55 \mathrm{~mm}$ \\
\hline $\mathrm{T}_{1}=\mathrm{S}_{1} \mathrm{C}_{1}$ & $5.40 \mathrm{gh}$ & $15.09 \mathrm{~g}$ & 39.91de & $31.80 \mathrm{~b}$ & $7.77 \mathrm{a}$ \\
\hline $\mathrm{T}_{2}=\mathrm{S}_{1} \mathrm{C}_{2}$ & $8.14 \mathrm{a}-\mathrm{d}$ & $21.82 \mathrm{ab}$ & $48.36 \mathrm{a}$ & $19.91 \mathrm{~g}$ & 3.391 \\
\hline $\mathrm{T}_{3}=\mathrm{S}_{1} \mathrm{C}_{3}$ & 7.20 cde & $15.80 \mathrm{fg}$ & $42.14 \mathrm{a}-\mathrm{d}$ & $31.08 \mathrm{bc}$ & $5.35 \mathrm{fgh}$ \\
\hline $\mathrm{T}_{4}=\mathrm{S}_{1} \mathrm{C}_{4}$ & $4.09 \mathrm{I}$ & $14.85 \mathrm{~g}$ & $42.25 \mathrm{a}-\mathrm{d}$ & 30.84 bc & $6.43 \mathrm{efg}$ \\
\hline $\mathrm{T}_{5}=\mathrm{S}_{1} \mathrm{C}_{5}$ & $9.11 \mathrm{a}$ & $12.48 \mathrm{~h}$ & $41.97 \mathrm{a}-\mathrm{e}$ & $31.13 \mathrm{bc}$ & 6.81cde \\
\hline $\mathrm{T}_{6}=\mathrm{S}_{1} \mathrm{C}_{6}$ & $7.42 \mathrm{~b}-\mathrm{e}$ & $17.53 \mathrm{def}$ & $46.18 \mathrm{a}-\mathrm{d}$ & $22.72 \mathrm{efg}$ & $6.15 \mathrm{~cd}$ \\
\hline $\mathrm{T}_{7}=\mathrm{S}_{2} \mathrm{C}_{1}$ & $4.87 \mathrm{hi}$ & $17.52 \mathrm{def}$ & $43.41 \mathrm{a}-\mathrm{d}$ & $31.63 \mathrm{~b}$ & $4.35 \mathrm{ijk}$ \\
\hline $\mathrm{T}_{8}=\mathrm{S}_{2} \mathrm{C}_{2}$ & $8.98 \mathrm{a}$ & $21.57 \mathrm{ab}$ & $46.56 \mathrm{abc}$ & $20.95 \mathrm{~g}$ & $3.74 \mathrm{kl}$ \\
\hline $\mathrm{T}_{9}=\mathrm{S}_{2} \mathrm{C}_{3}$ & $5.72 \mathrm{fgh}$ & $16.34 \mathrm{efg}$ & $47.47 \mathrm{ab}$ & $25.69 \mathrm{def}$ & $6.27 c-f$ \\
\hline $\mathrm{T}_{10}=\mathrm{S}_{2} \mathrm{C}_{4}$ & $7.05 \mathrm{de}$ & $18.26 \mathrm{def}$ & 40.29 cde & $28.61 \mathrm{bcd}$ & $6.95 \mathrm{bc}$ \\
\hline $\mathrm{T}_{11}=\mathrm{S}_{2} \mathrm{C}_{5}$ & $5.40 \mathrm{gh}$ & $9.44 \mathrm{i}$ & $36.10 \mathrm{e}$ & $47.58 \mathrm{a}$ & 4.96 hij \\
\hline $\mathrm{T}_{12}=\mathrm{S}_{2} \mathrm{C}_{6}$ & $8.06 \mathrm{a}-\mathrm{d}$ & $22.43 \mathrm{a}$ & $48.24 \mathrm{a}$ & $19.76 \mathrm{~g}$ & $3.24 \mathrm{jkl}$ \\
\hline $\mathrm{T}_{13}=\mathrm{S}_{3} \mathrm{C}_{1}$ & $5.88 \mathrm{fgh}$ & $17.66 \mathrm{def}$ & 40.40 cde & 26.59 cde & $6.25 \mathrm{def}$ \\
\hline $\mathrm{T}_{14}=\mathrm{S}_{3} \mathrm{C}_{2}$ & $6.63 \mathrm{ef}$ & $18.53 \mathrm{cde}$ & $47.07 \mathrm{ab}$ & $23.08 \mathrm{efg}$ & $6.2 \mathrm{c}-\mathrm{f}$ \\
\hline $\mathrm{T}_{15}=\mathrm{S}_{3} \mathrm{C}_{3}$ & $6.48 \mathrm{efg}$ & $15.96 \mathrm{fg}$ & $42.85 \mathrm{a}-\mathrm{d}$ & $29.27 \mathrm{bcd}$ & $8.18 \mathrm{a}$ \\
\hline $\mathrm{T}_{16}=\mathrm{S}_{3} \mathrm{C}_{4}$ & $8.57 \mathrm{ab}$ & $19.50 \mathrm{bcd}$ & $41.25 \mathrm{~b}-\mathrm{e}$ & $27.22 \mathrm{~b}-\mathrm{e}$ & $6.83 \mathrm{ab}$ \\
\hline $\mathrm{T}_{17}=\mathrm{S}_{3} \mathrm{C}_{5}$ & $9.10 \mathrm{a}$ & $22.40 \mathrm{a}$ & $42.30 \mathrm{a}-\mathrm{d}$ & $21.44 \mathrm{fg}$ & $4.76 \mathrm{ghi}$ \\
\hline $\mathrm{T}_{18}=\mathrm{S}_{3} \mathrm{C}_{6}$ & $8.33 \mathrm{abc}$ & $20.85 \mathrm{abc}$ & $45.45 \mathrm{a}-\mathrm{d}$ & $21.63 \mathrm{fg}$ & $4.93 \mathrm{fgh}$ \\
\hline CV\% & 4.81 & 4.10 & 4.53 & 5.02 & 7.00 \\
\hline $\begin{array}{l}\text { Level of } \\
\text { significance }\end{array}$ & $* *$ & $*$ & $* *$ & $*$ & $*$ \\
\hline
\end{tabular}

${ }^{*} \&^{* *}$ indicate significance at $5 \% \& 1 \%$ level of probability. Data were analyzed by transforming it in arcsine. "S" and "C" indicate seed size (large- $\mathrm{S}_{1}$, medium- $\mathrm{S}_{2}$, and small- $\mathrm{S}_{3}$ ) and cutting methods (half longitudinal- $\mathrm{C}_{1}$, half distal- $\mathrm{C}_{2}$, half proximal- $\mathrm{C}_{3}, 1 / 4^{\text {th }}$ crosswise- $\mathrm{C}_{4}, 1 / 4^{\text {th }}$ longitudinal- $\mathrm{C}_{5}$, whole tuber- $\mathrm{C}_{6}$ ), respectively

Small sized tubers ( $<20 \mathrm{~mm}$ grade) are less desired because of the low market price, while medium sized tubers $(28-40 \mathrm{~mm})$ are the most desired ones for its seed value and higher market price. Large sized tubers $(>40 \mathrm{~mm}$ ) have low consumer demand, but desired for industrial use and export. Therefore, the results indicate that distal half cut piece of large and medium size tuber are comparable to whole tuber of large size for obtaining high market value. Sultana and Siddique (1991) reported that the cut seed piece had significant influence on the number of main stems, yield of tubers per hill and yield of tubers per hectare. The highest yield of tubers (30.4 $\mathrm{t} / \mathrm{ha}$ ) was obtained from the largest seed piece (35gm) planted at $50 \mathrm{~cm} \times 22.5 \mathrm{~cm}$ spacing. Singh (1993) reported that potato tuber weighing 50gm were cut longitudinally into two pieces of approximately $25 \mathrm{gm}$ so that each had at least two prominent eyes and used as seed in different cutseed treatments. The inter-row spacing was kept constant at $60 \mathrm{~cm}$ but intra-row spacing for whole seed tubers was kept as per treatments of seed rate of 2, 3 or $4 \mathrm{t} / \mathrm{ha}$. And for cut tubers the intra-row spacing was reduced to half as compared to whole tubers. Average total yield was higher from whole tubers (14.3t/ha) than cut tubers (11.2t/ha) and it increased with planting rate. Net income was highest when whole seed tubers were planted at $4 \mathrm{t} / \mathrm{ha}$; this treatment also gave the highest yield of tubers suitable for seed (25-75gm). Hoque (2001) reported that cut tubers having single eye and cut tubers having at least two eyes planted six different spacing, to find the effect of different size of cut tubers on yield and to determine the optimum spacing for cut tubers and profitability of potato. Seed tubers having at least two eyes produces more yield than single eye. Maximum economic return may also be obtained from seed tubers having at least two eye pieces with a spacing of $40 \mathrm{~cm} \times 15$ $\mathrm{cm}$ (Benefit-Cost ratio $=3.15$ ). 

Table 5. Partial budget analysis of potato for different cutting methods and seed size with same spacing.

\begin{tabular}{|c|c|c|c|c|c|c|c|c|c|c|c|}
\hline \multirow[b]{2}{*}{ Treatment } & \multicolumn{4}{|c|}{ Labour hour/ha } & \multirow[b]{2}{*}{$\begin{array}{l}\text { Men } \\
\text { Days }\end{array}$} & \multirow{2}{*}{$\begin{array}{c}\text { Total } \\
\text { labour } \\
\text { Cost } \\
\text { (Tk.) } \\
\end{array}$} & \multirow[b]{2}{*}{$\begin{array}{l}\text { Seed } \\
\text { Cost } \\
\text { (Tk.) } \\
\end{array}$} & \multirow{2}{*}{$\begin{array}{c}\text { Total } \\
\text { Variable } \\
\text { Cost (Tk.) }\end{array}$} & \multirow{2}{*}{$\begin{array}{c}\text { Gross } \\
\text { Return } \\
\text { (Tk./ha) } \\
\end{array}$} & \multirow[b]{2}{*}{$\begin{array}{c}\text { Net } \\
\text { Benefit } \\
\text { (Tk./ha) }\end{array}$} & \multirow[b]{2}{*}{$\begin{array}{c}\text { Benefit } \\
\text { Cost } \\
\text { Ratio } \\
\end{array}$} \\
\hline & $\begin{array}{c}\text { Seed } \\
\text { cutting }\end{array}$ & $\begin{array}{c}\text { Fertilizer } \\
\text { application/ } \\
\text { Spraying } \\
\end{array}$ & Harvesting & Total & & & & & & & \\
\hline $\mathrm{T}_{1}=\mathrm{S}_{1} \mathrm{C}_{1}$ & 300 & 90 & 430 & 820 & 102 & 10,200 & 51,200 & 61,400 & $2,70,116$ & $20,87,16$ & 3.39 \\
\hline $\mathrm{T}_{2}=\mathrm{S}_{1} \mathrm{C}_{2}$ & 310 & 90 & 430 & 830 & 104 & 10,400 & 65,600 & 76,000 & $3,34,286$ & $2,58,286$ & 3.39 \\
\hline $\mathrm{T}_{3}=\mathrm{S}_{1} \mathrm{C}_{3}$ & 310 & 90 & 430 & 830 & 104 & 10,400 & 60,000 & 70,400 & $2,39,265$ & $1,68,865$ & 2.99 \\
\hline $\mathrm{T}_{4}=\mathrm{S}_{1} \mathrm{C}_{4}$ & 250 & 89 & 425 & 764 & 96 & 9,600 & 33,400 & 43,000 & $2,52,843$ & $2,09,843$ & 4.83 \\
\hline $\mathrm{T}_{5}=\mathrm{S}_{1} \mathrm{C}_{5}$ & 260 & 89 & 425 & 774 & 97 & 9,700 & 32,200 & 41,900 & $2,55,197$ & $2,13,297$ & 5.09 \\
\hline $\mathrm{T}_{6}=\mathrm{S}_{1} \mathrm{C}_{6}$ & - & 92 & 427 & 519 & 65 & 6,500 & $1,15,600$ & $1,22,100$ & $3,76,555$ & $2,54,455$ & 2.08 \\
\hline $\mathrm{T}_{7}=\mathrm{S}_{2} \mathrm{C}_{1}$ & 290 & 89 & 428 & 807 & 101 & 10,100 & 46,000 & 56,100 & $2,43,761$ & $1,87,661$ & 3.34 \\
\hline $\mathrm{T}_{8}=\mathrm{S}_{2} \mathrm{C}_{2}$ & 290 & 89 & 428 & 807 & 101 & 10,000 & 43,470 & 53,470 & $2,64,789$ & $2,11,319$ & 3.95 \\
\hline $\mathrm{T}_{9}=\mathrm{S}_{2} \mathrm{C}_{3}$ & 260 & 89 & 428 & 777 & 97 & 9,700 & 43,470 & 53,170 & $2,50,956$ & $1,97,786$ & 3.71 \\
\hline $\mathrm{T}_{10}=\mathrm{S}_{2} \mathrm{C}_{4}$ & 270 & 87 & 420 & 777 & 97 & 9,700 & 23,000 & 32,700 & $1,50,910$ & $1,18,210$ & 3.61 \\
\hline $\mathrm{T}_{11}=\mathrm{S}_{2} \mathrm{C}_{5}$ & 290 & 87 & 420 & 798 & 100 & 10,000 & 20,470 & 30,470 & $1,21,616$ & 91,146 & 2.99 \\
\hline $\mathrm{T}_{12}=\mathrm{S}_{2} \mathrm{C}_{6}$ & - & 90 & 425 & 515 & 64 & 6,400 & 69,000 & 75,400 & $3,47,586$ & $2,72,186$ & 3.60 \\
\hline $\mathrm{T}_{13}=\mathrm{S}_{3} \mathrm{C}_{1}$ & 290 & 87 & 420 & 798 & 100 & 10,000 & 16,750 & 26,750 & $2,06,143$ & $1,79,393$ & 6.70 \\
\hline $\mathrm{T}_{14}={ }_{3} \mathrm{C}_{2}$ & 305 & 87 & 420 & 812 & 101 & 10,100 & 16,750 & 26,850 & $2,45,126$ & $2,18,276$ & 8.13 \\
\hline $\mathrm{T}_{15}=\mathrm{S}_{3} \mathrm{C}_{3}$ & 305 & 87 & 420 & 812 & 101 & 10,100 & 25,000 & 35,100 & $2,01,685$ & $1,66,585$ & 4.74 \\
\hline $\mathrm{T}_{16}=\mathrm{S}_{3} \mathrm{C}_{4}$ & 265 & 85 & 415 & 765 & 96 & 9,600 & 8,250 & 17,850 & 94,273 & 76,423 & 4.28 \\
\hline $\mathrm{T}_{17}=\mathrm{S}_{3} \mathrm{C}_{5}$ & 275 & 85 & 415 & 775 & 97 & 9,700 & 11,000 & 20,700 & 98,914 & 78,214 & 3.78 \\
\hline $\mathrm{T}_{18}=\mathrm{S}_{3} \mathrm{C}_{6}$ & - & 89 & 420 & 509 & 63 & 6,300 & 47,250 & 53,550 & $3,14,173$ & $2,60,623$ & 4.86 \\
\hline
\end{tabular}

Labour cost=Tk. 100. 1 man day= 8 hour. Seed price= 25/-per kg ( small size), 23/-per kg ( medium size). 20/-per kg (large size). $S_{1}, S_{2}, S_{3}$ indicates large, medium and small size tubers. "S" and " $\mathrm{C}$ " indicate seed size (large- $\mathrm{S}_{1}$, medium- $\mathrm{S}_{2}$, and small- $\mathrm{S}_{3}$ ) and cutting methods (half longitudinal- $\mathrm{C}_{1}$, half distal- $\mathrm{C}_{2}$, half proximal- $\mathrm{C}_{3}, 1 / 4^{\text {th }}$ crosswise- $\mathrm{C}_{4}, 1 / 4^{\text {th }}$ longitudinal- $\mathrm{C}_{5}$, whole tuber- $\mathrm{C}_{6}$ ), respectively 


\subsection{Economic analysis}

In respect of cutting methods higher labour cost was involved compared to whole tuber. The highest cost of production was Tk.1, 22,100/ha. and gross return was Tk. $3,76,555 /$ ha in $\mathrm{T}_{6}$ followed by $T_{2}$ where the cost of production was Tk. 76,000 and gross return was Tk. 3,34,286/ha (Table 5). Despite the highest gross return (Tk. $3,76,555 /$ ha.), high variable cost (Tk. 1, 22,100) reduced net benefit (Tk. 2, 54,455) and BCR (2.08) in case of large whole tubers $\left(\mathrm{T}_{6}\right)$. The highest net benefit (Tk. 2, 72,186) was calculated from $\mathrm{T}_{12}$ due to higher gross return (Tk. 3, 47,586) and lower variable cost (Tk. 75,400). Different cutting methods influenced the BCR of potato. It was evident that the highest net return (Tk. 2, 72,186) was found from $\mathrm{T}_{12}$ followed by $\mathrm{T}_{18}$ (Tk. 2, 60,623), $\mathrm{T}_{2}$ (Tk. 2, 58,286/ha), $\mathrm{T}_{6}$ (Tk. 2, 54,455/ha) and the highest BCR was found from $\mathrm{T}_{14}(8.13)$.

\section{Conclusions}

In consideration of producing higher plant height, foliage coverage, no. of stems/hill, no. of tubers/hill, per hectare yield, the large seed size tubers were better than those of smaller ones. Whole tubers performed better in relation to plant height foliage coverage, no. of stems per hill, no. of tubers per hill, per hectare yield than cut tubers, while the half cut distal $\left(\mathrm{C}_{2}\right)$ showed better performance than proximal end. Half cut distal parts found the best for producing the marketable tubers. Large whole tubers proved to be the best for maximum vegetative growth, tuber yield/plant and per hectare yield, while large half cut distal parts was the next. Medium whole tubers $\left(T_{12}\right)$ proved to be the best in case of net returns /hectare followed by small whole tubers $\left(\mathrm{T}_{18}\right)$ and large half cut longitudinal $\left(\mathrm{T}_{2}\right)$. Small half cut distal $\left(\mathrm{T}_{14}\right)$ was found to be the best regarding benefit cost ratio (8.13) followed by the small half longitudinal (6.7), large half cut longitudinal (5.09) and small whole tubers (4.86). Considering all the aspects, it was concluded that $\mathrm{T}_{5}$ from large sized tuber, $\mathrm{T}_{8}$ from medium sized and $T_{14}(1 / 2$ cut distal $)$ from small sized tuber could be the optimum for planting cut tubers.

\section{References}

Anonymous, 2010. Annual Report, 2009-10. Tuber Crops Research Centre, BARI, Joydebpur, Gazipur 1701. 202 p

Banarjee, M. K., Samdyan, J. S. Hooda, R. S. and Tong, D. S. 1988. Effect of cut seed size, gibberellic acid and spacing on growth and yield of seed potato cv. Kufri Chandramukhi. Haryana Journal of Agronomy, 4(2): 128-130.

Bushan, A. J., Mitchell, P. D., Copas, M. E., Drillias, M. J.2007. Evaluation of the effect of density on potato yield and tuber size distribution. Crop Science, 47(6): 2462-2472.

Chandla, V. K., Shiv, K., Singh, M. N., Verma, K. D., Khurana, S. M. P., Kumar, S. and Pandey, S. K. 2001. Role of aphids in degeneration of seed stocks in the higher hills. Journal of the Indian Potato Association, 28(1): 117-118.

Garba, A., Udom, G. N., Ikeasomba, M. A., Hasuruma, A. 2005. Influence of seed size and planting date on the growth, development and yield of potato varieties in Bauchi. Journal of Agricultural Science, 4(1): 19-22.

Hoque, M. A. 2001. Effect of cut seed size and spacing on the yield and profitability of potato. Annual report on post flood rehabilitation and adaptive research support project, BARI, Munshiganj, $17 \mathrm{p}$.

Khurana, S. C., Dhankhar, B. S. and Shekhawat, G. S. 1994. Effect of seed rate and seed size on potato hybrid JH 222. Potato: present and future. Proceedings of the national symposium held on Modipuran during 1-3, March, 1993. 164-167 pp.

Nandekar, D. N. and Dubey, K. C. 1993. Potato yield and its economics as influenced by cut and whole tuber seed. Journal of Indian Potato Association, 20(2): 26. 
Shaha, A., Das, B., and Roy, A. K. S. 2004. Effect of spacing and seed size on growth and yield of tuberlets of TPS of potato under terai agro-climatic situation of West Bengal. Indian Agriculturist, 48(1/2): 141144.

Sultana, N. and A. Siddique. 1991. Effect of cut seed piece and plant spacing on the yield and profitability of potato. Bangladesh Horticulture, 19(1): 37-43.

Singh, N. 1993. Effect of cut and whole seed tubers and seed rate on the emergence, yield and economics of potato in the
Nilgiri hills. Journal of Indian Potato Association, 20(3-4): 260-262.

Talukder, M. Z. 2004. Role of BADC in development of quality seed potato in private sector. Compendium on Seed Potato production, Published by SID / DANIDA, Dhaka, Bangladesh.

Zebarth, B. J., Arsenault,W. J., Sanderson, J. B. 2006. Effect of seed piece spacing and nitrogen fertilization of tuber yield components, and nitrogen use efficiency parameters of two potato cultivars. American Journal of Potato Research, 83(4): 289-296. 
